\title{
From Type II Upconversion to SPDC: A Path to Broadband Polarization Entanglement in Poled Fibers
}

\author{
E.Y. Zhu ${ }^{1}$, L.G. Helt ${ }^{2}$, M. Liscidini ${ }^{2}$, L. Qian ${ }^{1}$, J.E. Sipe ${ }^{2}$, A. Canagasabey ${ }^{3}$, C. Corbari ${ }^{3}$, M. Ibsen ${ }^{3}$, and \\ P. G. Kazansky ${ }^{3}$ \\ ${ }^{I}$ Dept. of Electrical and Computer Engineering, University of Toronto, 10 King's College Road, Toronto, Ontario, Canada M5S $3 G 4$ \\ ${ }^{2}$ Department of Physics, University of Toronto, 60 St. George St. Toronto Ontario, Canada M5S 1 A7 \\ ${ }^{3}$ Optoelectronics Research Centre, University of Southampton, SO17 1BJ, United Kingdom \\ AuthorE-mail: eric.zhu@utoronto.ca
}

\begin{abstract}
We report type II sum-frequency and second-harmonic generation in a 24-cm-long periodically-poled silica fiber. Quasi-phase matching is achieved for orthogonally-polarized signal and idler over $1520-1575 \mathrm{~nm}$, demonstrating the path to in-fiber broadband polarization-entangled photon pair generation.

(C)2009 Optical Society of America

OCIS codes: (270.0270) Quantum Optics, (190.4370) Nonlinear optics, fibers
\end{abstract}

\section{Introduction}

In-fiber generation of polarization-entangled photon pairs has been demonstrated in dispersion-shifted fiber [1] and photonic crystal fiber [2] by exploiting spontaneous four-wave mixing, a $\chi^{(3)}$ process. In both cases, the downconverted photons are co-polarized with the pump and polarization entanglement is only achieved through additional steps such as employing a Sagnac loop and polarizing beam splitter $[1,2]$.

An alternative approach is to use a $\chi^{(2)}$ process in periodically poled silica fibers (PPSF). This second-order nonlinearity arises from the presence of a DC poling field through the Kerr nonlinearity, $\chi_{i j k}^{(2)} \equiv 3 \chi_{i j k l}^{(3)} E_{l}^{D C}$. For convenience, we shall take this poling field to be oriented in the $x$-direction (see inset of Fig. 1a for our coordinate convention). Due to the isotropic nature of silica $\left(\chi_{x x x x}^{(3)}=3 \chi_{y y x x}^{(3)}=3 \chi_{y x y x}^{(3)}=3 \chi_{x y y x}^{(3)}\right)$, there are only four elements of the $\chi^{(2)}$ tensor we need to concern ourselves with in PPSF: $\chi_{x x x}^{(2)}, \chi_{y y x}^{(2)}, \chi_{y x y}^{(2)}$, and $\chi_{x y y}^{(2)}$.

From these four tensor elements, three SPDC processes arise in our poled fiber. The first two tensor elements $\left(\chi_{x x x}^{(2)}, \chi_{y y x}^{(2)}\right)$ correspond to copolarized signal and idler pairs (type I phase-matching), while orthogonally polarized signal and idler pairs (type II phase-matching) are achieved with the $\chi_{y x y}^{(2)}$ (or $\chi_{x y y}^{(2)}$ ) element. In the context of SPDC, the subscripts of the $\chi_{i j k}^{(2)}$ tensor refer to the idler $(i)$, signal $(j)$ and pump $(k)$ polarizations.

Although parametric fluorescence and correlated photon pair production have been demonstrated in periodically poled fibers [3], there has been no report of type II parametric generation. For an incident pump beam at an appropriate wavelength and polarized in the $y$ direction, the $\chi_{y x y}^{(2)}$ tensor element should lead to the direct generation of maximally polarization-entangled photons in PPSF via type II SPDC.

In this work, we experimentally demonstrate the presence of the $\chi_{y x y}^{(2)}$ tensor element in PPSF via continuous wave (CW) second-harmonic generation (SHG) and sum-frequency generation (SFG); with the latter, we also observe broadband quasi-phase-matching of the signal and idler from 1520-1575nm. Finally, based on the experimental SHG and SFG efficiencies, we estimate the value of $\chi_{y x y}^{(2)}$ and calculate the spectral brightness and bandwidth of the polarization-entangled photons that could be produced in this fiber using a y-polarized pump beam.

\section{Second Harmonic Generation Experiment}

In our experiment, the PPSF is a step index fiber with two large air-holes in the cladding (Fig. 1a). Poling of the fiber involves inserting two electrodes into the air-holes and applying a DC potential. Periodic UV erasure is subsequently performed on the poled fiber to enable quasi-phase-matching of CW-SHG for fundamental wavelengths $\lambda_{F} \sim 1.5 \mu \mathrm{m}[4]$.

We experimentally examine three SHG processes (Fig. 1b) in the fiber; two in which the fundamental beams are co-polarized $\left(\chi_{x x x}^{(2)}\right.$, and $\left.\chi_{x y y}^{(2)}\right)$, and a third (which yields a peak at $1545.6 \mathrm{~nm}$ ) in which we use cross-polarized fundamental beams $\left(\chi_{y x y}^{(2)}\right)$. The experimental setup is shown in Fig. 1a.

We are able to experimentally distinguish between all three processes (Fig. 1b); by our calculations, this can be explained in terms of a non-negligible fiber birefringence that allows for the phase-matching condition to be met at different wavelengths for different polarization configurations. 

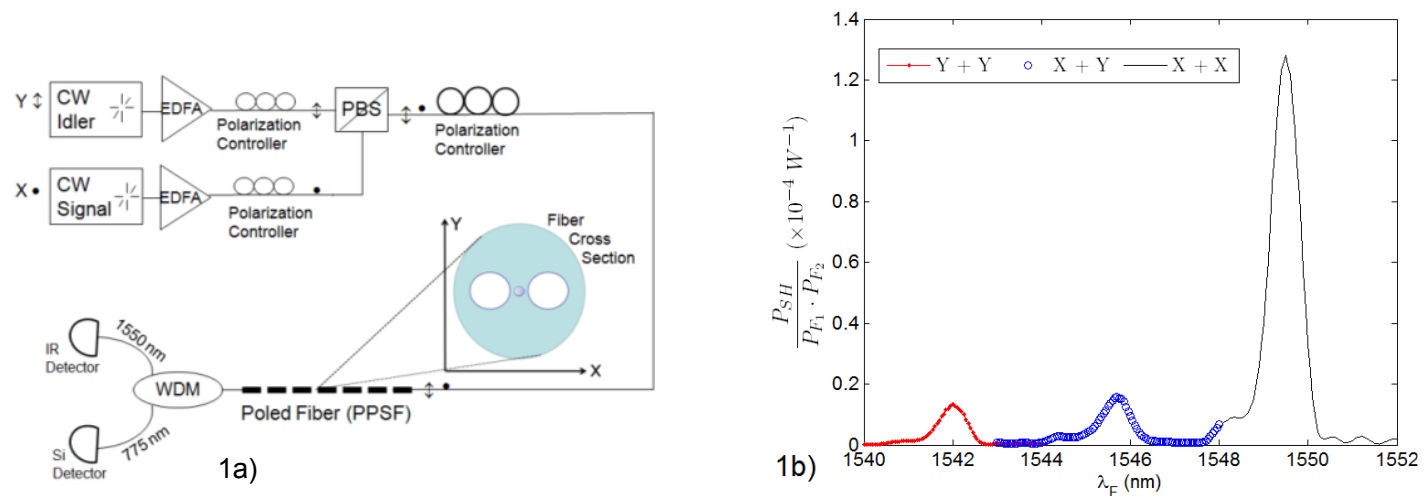

Fig.1a) Experimental setup of the SHG and SFG experiments with inset of fiber geometry of the poled fiber. Fig.1b) SHG efficiency as a function of the fundamental wavelength. The center peak in the SHG spectrum is produced by cross-polarized fundamental beams.

\section{Sum-Frequency Generation Experiment and SPDC Predictions}

To confirm the presence of a non-zero $\chi_{y x y}^{(2)}$ tensor element, we also carry out a type II sum-frequency generation experiment (Fig. 1a). Two CW beams, the signal being $x$-polarized and the idler $y$-polarized, are combined and incident upon the PPSF. By tuning the signal and idler wavelengths from 1520-1575 nm, a range limited by our equipment, we are able to obtain an SFG signal when $\lambda_{S F}$ is between $772-774 \mathrm{~nm}$ (Fig 2a), with a peak at $772.8 \mathrm{~nm}$.

The SHG and SFG spectra tell us that type I and type II SPDC should be phase-matched at different pump wavelengths - 775 and $771 \mathrm{~nm}$ for type I, and $772.8 \mathrm{~nm}$ for type II. Judicious pump wavelength selection relaxes the requirement for stringent pump polarization alignment. That is, even if a $772.8 \mathrm{~nm}$ incident pump field is not completely $y$-polarized, phase-matching conditions only allow for the generation of type II SPDC pairs in the PPSF.

We calculate the spectral brightness (Fig. 2b) of type II continuous-wave SPDC (the pump is $y$-polarized) in our fiber by applying the formalism from [5] and using the value $\chi_{y x y}^{(2)}=0.012 \mathrm{pm} / \mathrm{V}$ determined from our SHG experiment. Fig. $2 \mathrm{~b}$ suggests that the type II SPDC should be at least $60 \mathrm{~nm}$ in bandwidth, and separation of signal and idler can be achieved by detuning the pump to the red without decreasing the spectral brightness. Finally, we calculate that the total SPDC flux could be as high as $4 \times 10^{5}$ pairs per second per $\mathrm{mW}$ of $\mathrm{CW}$ pump power.
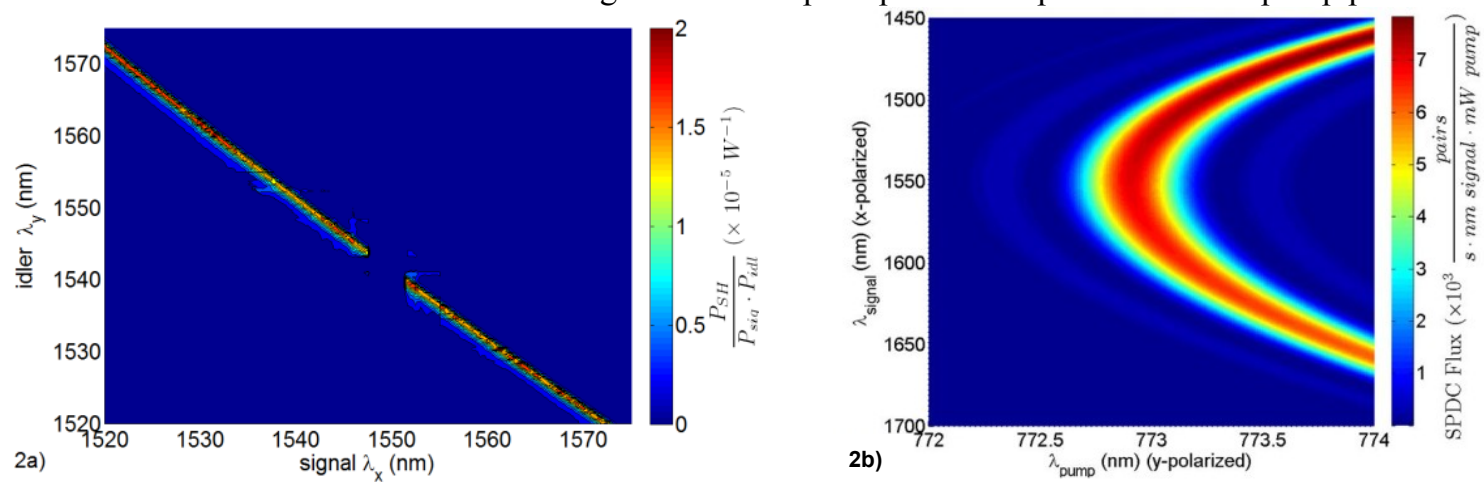

Fig. 2a) Experimental SFG spectrum showing that the SFG spectrum peaks for $\lambda_{S H} \sim 773 \mathrm{~nm}$. Data where the co-polarized SHG is strong $\left(\lambda_{y} \sim 1542 \mathrm{~nm}, \lambda_{x} \sim 1549.6 \mathrm{~nm}\right)$ are removed. Fig.2b) Theoretical SPDC tuning curve for varying pump wavelengths.

\section{Conclusions \& Outlook}

We experimentally demonstrate the presence of the $\chi_{y x y}^{(2)}$ tensor element in PPSF through SHG and SFG. Our calculations suggest that the total type II SPDC flux produced to be upwards of $4 \times 10^{5} \frac{\mathrm{pairs}}{\mathrm{s} \cdot \mathrm{mW}}$; we also expect the SPDC spectrum to be broadband, which is supported by our experimental SFG spectrum. By exploiting this tensor, maximally polarization-entangled photon pairs can be produced directly in-fiber.

\section{References}

[1] J. Chen, K.F. Lee, C. Liang, and P. Kumar, Opt. Lett. 31, 2798-2800 (2006)

[2] J. Fan, M. D. Eisaman, and A. Migdall, Opt. Express 15, 18339-18344 (2007)

[3] K. Phan Huy, et al., Opt. Express 15, 4419-4426 (2007)

[4] A. Canagasabey, C. Corbari, Z. Zhang, P.G. Kazansky, and M. Ibsen, Opt. Lett. 32, 1863-1865 (2007)

[5] Z. Yang, M. Liscidini, and J. E. Sipe., Phys. Rev. A 77, 033808 (2008) 